\title{
Combination Therapy
}

\author{
MARGO A. DENKE, MD, FACP, FACE, FAHA
}

\begin{abstract}
Summary
Adult Treatment Panel III (ATP III) guidelines recommend specific treatment targets for low-density lipoprotein cholesterol (LDL-C) levels according to an individual's short-term and long-term risk for coronary heart disease (CHD). Therapeutic lifestyle changes are recommended for all patients at any level of risk for $\mathrm{CHD}$. Although most patients will achieve some LDL-C lowering with lifestyle modification, ATP III recognizes that a majority of patients with dyslipidemia will require drug therapy to reach their LDL goal. Surveys of physicians' practices suggest that only a small percentage of patients enrolled in an active treatment program actually achieve their LDL-C target. In addition, other surveys suggest that not all patients who are treatment candidates are receiving assessment. From a medication perspective, either up-titration of statin dose or the use of drug combinations should further enhance the likelihood of achieving target lipid levels. Combination therapies that target both the endogenous and exogenous pathways of cholesterol synthesis are particularly attractive. This paper reviews the pharmacotherapeutic effects of combination therapy, summarizes the strengths and weaknesses of current lipid-lowering drug combinations, and identifies the potential contribution of the novel cholesterol absorption inhibitor, ezetimibe, to the LDL-C treatment algorithm.
\end{abstract}

KEWORDS: Cholesterol, Combination therapy, Dyslipidemia, LDL-C

\section{Author Correspondence}

MARGO A. DENKE, MD, FACP, FACE, FAHA, Professor of Medicine, University of Texas Southwestern Medical Center at Dallas, 5323 Harry Hines Blvd., Dallas, TX 75390-9052. Tel: (214) 648-3111; Fax: (214) 648-7150; E-Mail: Margo.Denke@UTSouthwestern.edu

Copyright $\odot$ 2003, Academy of Managed Care Pharmacy. All rights reserved.
S imilar to the prescription practices in the treatment of hypertension, combination therapies are often required for optimal lipid management. Despite the fact that combination therapy can be effective, well tolerated, and safe, surveys suggest that only a few patients are receiving combination therapies for dyslipidemia. For patients, combination therapy may offer the only effective means to achieve desired lipid targets. As with the treatment of hypertension, the combination of 2 low-dose drugs can achieve lipid reductions that exceed those observed with high-dose monotherapy. When combination therapy employs 2 different drug classes with complementary mechanisms of action, the effectiveness is additive. Achieving target low-density lipoprotein cholesterol (LDL-C) goals using a combination that includes a statin can be particularly effective. Some combinations may prove to be better tolerated than high-dose monotherapy with statins, particularly when the lipid-lowering capacity of the add-on drug allows for reduction in the dose of the original therapy and offers a favorable side-effect profile. Examples of currently available combination therapies are described below.

\section{Currently Available Combination Therapies}

Statins are identified as the drug of first choice for lowering of LDL-C by Adult Treatment Panel III (ATP III). Consequently, the most logical combination strategy would be to add a drug from another class to statin therapy. Combination therapy with statins has some distinct advantages. In general, lower doses of each drug can be used, minimizing the risk of side effects. Additionally, combining a drug with a mechanism of action different from that of a statin typically achieves at least an additional $10 \%$ reduction in LDL-C, whereas doubling the dose of a statin will achieve only a $6 \%$ additional lowering of LDL-C. ${ }^{1}$ Bile acid resins and niacin are common add-on therapy to a statin. Other statin combinations, while effective in favorably modifying lipid profiles, must be used with caution due to an increased possibility of side effects.

Statins and bile acid resins are the most common combination. Adding a statin to bile acid resin monotherapy overcomes the compensatory increase in cholesterol synthesis that occurs during bile acid resin therapy. This combination has been shown to reduce LDL-C by as much as 50\%, an amount equivalent to that achieved by high-dose statin alone. ${ }^{1}$ The inconvenience of multiple daily dosing of bile acid resins and their accompanying GI side effects limits the effectiveness of these agents in either monotherapeutic or combination regimens.

The combination of low-dose statins and fibrates appears to have complementary effects on triglycerides and LDL-C and proves to be especially attractive for patients with mixed hyperlipidemia characterized by elevated triglyceride and LDL-C. However, caution must be exercised when using statins and fibrates together because this combination has an increased risk of myopathy beyond what is normally observed with either drug 


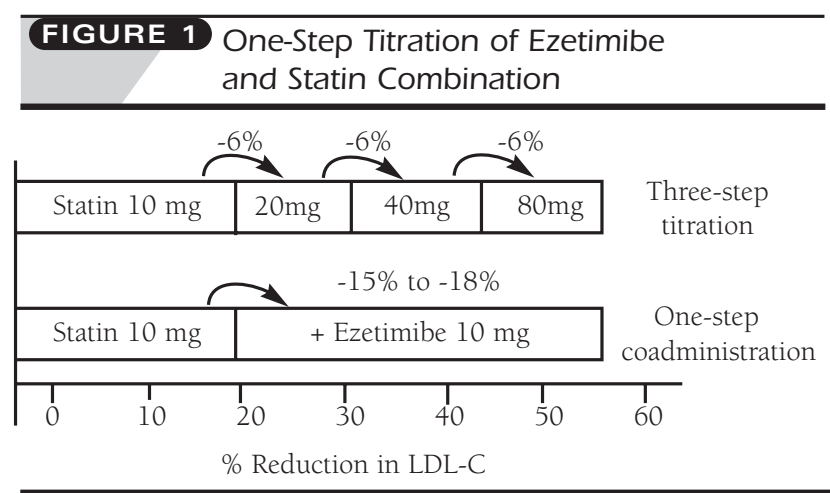

class alone. Appropriate precautions, such as using the combination with the lowest effective doses in patients who have normal liver and kidney function, been educated regarding the signs and symptoms of myopathy, and been instructed to stop drugs if symptoms occur, should help minimize the risk. Some research has suggested that the combination of fenofibrate and statin is associated with a lower rate of myopathy than gemfibrozil and statin. Given the low incidence of myopathy, however, far larger studies would be required to confirm this observation. ${ }^{2}$

The combination of statin and niacin is an effective combination for patients with mixed hyperlipidemia as well as for patients with simple hypercholesterolemia. For example, 2 grams of niacin added to a stable dose of statin achieved an additional 31\% LDL-C lowering. ${ }^{3}$ Unfortunately, similar to the combination of fibrates and statins, the combination of niacin and statin is associated with an increased risk of myopathy. Similar to bile acid resins, niacin as a single agent or as part of combination therapy is associated with vasodilatory side effects that are difficult for some patients to tolerate. Additional notes of caution are required-niacin can unmask glucose intolerance and worsen glycemic control in patients with diabetes; niacin can raise uric acid levels and precipitate an attack of gout.

Not all combination therapies employ the use of a statin. The combination of bile acid resins and nicotinic acids was used extensively before statins were even developed. This combination achieves LDL-C reductions of $32 \%$ to $43 \%$ with high-density lipoprotein cholesterol (HDL-C) increases of $37 \%$ to $43 \% .{ }^{4}$ The use of this combination is limited by the gastrointestinal and vasodilatory side effects of the drugs. Lowering the dose of each drug can reduce the side effects and achieve reasonably good improvement in LDL-C and HDL-C, although improvement is not as significant as with higher doses. ${ }^{4}$

Although used infrequently, the fibrate-niacin combination may have some synergy with respect to the individual effects of the drugs on triglyceride and HDL-C levels and may prove useful in patients with very high triglyceride levels. ${ }^{5}$ The addition of fish oil supplements can also be employed to lower triglyceride levels.

\section{Contribution of Cholesterol Absorption Inhibitors} to Combination Therapy

Several alternatives to currently available combination therapies are in development. Ezetimibe, the first of a new class of selective cholesterol absorption inhibitors, was recently approved by the U.S. Food and Drug Administration; it offers a mechanism of action distinct from statins and other agents. Cholesterol absorption inhibitors significantly reduce intestinal absorption of both dietary and biliary cholesterol from the intestine. The combination of ezetimibe and a low-dose statin results in a decrease of the absorption of cholesterol from the intestine as well as a decrease in the synthesis of cholesterol in the body. The net effect is an up-regulation of LDL receptor activity in the liver and a reduction in plasma LDL concentrations. The additive nature of these 2 effects explains the synergy of the combination (Figure 1).

Phase 2 and 3 studies from the ezetimibe development program have demonstrated that combining selective cholesterol absorption inhibition with statins is an effective strategy for optimizing cholesterol-lowering effects. For example, subjects with hypercholesterolemia treated with simvastatin $10 \mathrm{mg}$ daily for 14 days achieved a 35\% reduction in LDL-C while those treated with simvastatin $10 \mathrm{mg}$ plus ezetimibe $10 \mathrm{mg}$ achieved a reduction of $52 \%$ - a decrease in LDL-C expected from a simvastatin dose of $80 \mathrm{mg}$. Additionally, ezetimibe has virtually no effect on statin pharmacokinetics. Thus, combining ezetimibe with a statin may reduce the dose of the latter drug required to achieve target levels, or, in patients who respond poorly to statins, may improve the lipid-lowering effect.

A similar phase 2 coadministration study found that the decrease in LDL-C was 40\% for $10 \mathrm{mg}$ atorvastatin alone, $23 \%$ for $10 \mathrm{mg}$ ezetimibe alone, and 56\% for both drugs given together. Consistent with the findings of the simvastatin coadministration studies, the additional reduction in LDL-C cholesterol seen with the combination of $10 \mathrm{mg} /$ day ezetimibe and $10 \mathrm{mg} /$ day atorvastatin was equivalent to the reduction expected with $80 \mathrm{mg} /$ day atorvastatin monotherapy. ${ }^{7}$

\section{Conclusion}

For the majority of patients with dyslipidemia, statins remain the treatment of choice. Despite their efficacy, evidence from clinical trials and clinical practice demonstrates that the majority of dyslipidemic patients do not achieve ATP III target LDL-C goals. This is especially true for high-risk patients where lower LDL targets are recommended. Multiple factors contribute to the failure to achieve lipid goals with statin therapy. Some failures occur because highrisk patients who are excellent candidates for therapy are not identified. Other failures occur because the prescribed dose of statin is insufficient to achieve the recommended LDL lowering. For the most part, statins have been proven to be exceptionally safe drugs. However, some patients clearly experience side effects such as muscle pain and elevated liver enzymes, particularly at higher doses. If high doses of statins are poorly tolerated, niacin and bile acid resins are typical alternative or add-on agents. The use of 
these agents is limited by their side-effect profile. Ezetimibe is a promising new alternative in phase 3 development. Ezetimibe holds the potential to become a viable agent for either monotherapy or as an add-on to a statin.

\section{DISCLOSURES}

Dr. Danke received an honorarium for participating in the symposium on which this article is based. She serves as a speaker for Merck and Co., Inc.; Merck/Schering-Plough; and Roche.

\section{REFERENCES}

1. Witzum JL. Drugs used in the treatment of hyperlipoproteinemias. In: Hardman JG, Limberg JE, eds. Goodman and Gilman's The Pharmacologic Basis of Therapeutics. 9th ed. New York, NY: McGraw-Hill; 1996:875-97.

2. Illingworth DR. Management of hypercholesterolemia. Med Clin North Am. $2000 ; 84: 23-42$
3. Wolfe ML, Vartanian SF, Ross JL, et al. Safety and effectiveness of Niaspan when added sequentially to a statin for treatment of dyslipidemia. Am J Cardiol. 2001;87:476-79

4. McKenney JM. Improving cholesterol control in managed care populations. Am J Managed Care. 2000;6(suppl.):S997-S1007.

5. Rubins HB, Robins SJ, Collins SD, et al, for the Veterans Affairs HighDensity Lipoprotein Cholesterol Intervention Trial Study Group. Gemfibrozil for the secondary prevention of coronary heart disease in men with low levels of high-density lipoprotein cholesterol. N Eng J Med. 1999;341:410-18.

6. Kosoglou T, Meyer I, Musiol B, et al. Pharmacodynamic interaction between the new selective cholesterol absorption inhibitor SCH 58235 and simvastatin [abstract]. Atherosclerosis. 2000;151:135.

7. Kosoglou T, Seiberling M, Statkevich P, et al. Pharmacodynamic interaction between the new selective cholesterol absorption inhibitor ezetimibe and atorvastatin [abstract]. J Am Coll Card. 2001;37(2 suppl A):229. 